American Journal of Infectious Diseases 4 (2): 168-173, 2008

ISSN 1553-6203

(C) 2008 Science Publications

\title{
Central Nervous System Tuberculomas: A Review Article
}

\author{
Mutasem Abuhamed, Xiao Bo and Cai Yan \\ Department of Neurology, The First Affiliated Xiangya Hospital, \\ Central South University, Changsha, Hunan, People's Republic of China
}

\begin{abstract}
Central Nervous System Tuberculomas (CNSTs), the most dangerous form of tubercolomas, remains a public health problem, particularly in the developing country. Central nervous system tuberculomas, either single or multiple, usually presents as a diagnostic challenge because it resembles many other infectious and non-infectious medical conditions, particularly in patients without constitutional symptoms or evidence of tuberculosis elsewhere in the body. Here we reviewed the available evidence to answer some of unanswered questions, especially those on the diagnosis and treatment of Central Nervous system tuberculomas. The clinical symptomatology of intracranial tuberculoma was not specific. The CSF profile may be completely normal, even in repeated specimen's.The MRI was superior to the CT scan in showing not only the location and diagnostic features of tuberculoma, but also in detecting oedema of the brain. Surgical intervention in situation of acute complication of CNSTs, when there was no response to chemotherapy. Prompt diagnosis of Central nervous system tuberculomas result in early treatment and better outcome.
\end{abstract}

Key words: CNSTs, solitary tuberculomas, antituberculosis drugs

\section{INTRODUCTION}

Tuberculosis is a highly widespread disease in developing countries. Meningitis is by far the most frequent manifestation of tuberculosis in the central nervous system ${ }^{[1]}$. However, intracranial masses remain an unusual presentation of such infection that usually affects immunocompromised patients. Central Nervous System Tuberculomas (CNST), either multiple or single, usually presents as a diagnostic challenge because it has a similar appearance to many other noninfectious infectious and infectious medical conditions, specifically in patients without constitutional symptoms or evidence of tuberculosis elsewhere in the body ${ }^{[1-4]}$. Approximately $1 \%$ of tuberculosis patients develop CNST. Between 1910 and 1931, 34\% of intracranial masses in the USA and Europe were tuberculomas. In later clinical sequence from the same regions the ratio fell to less than $4 \%$. But in developing countries CNST still constitute one-third of intracranial intracranial masses $^{[5-7]}$. However, in many parts of the world CNST is a rather common neurological disorder that results from hematogenous spread of a distant focus of Mycobacterium tuberculosis infection. Intracranial Tuberculomas are usually solitary lesions, although 15- 34\% are multiple ${ }^{[7]}$. The diagnosis and management of CNST is an important public health problem in both developing and industrialised nations. Dissimilar pulmonary tuberculosis, which has been the subject of many clinical and experimental studies, the diagnosis and treatment of CNST have received slight consideration. How the disease kills or disables most of those it infects is not clear; the best diagnostic tests are disputable; the most efficient choice, dose and treatment duration of antituberculosis drugs are not known; and the result from chemotherapy and neurosurgical intervention has been difficult to study.

\section{DIAGNOSIS OF CNSTs}

The diagnosis and treatment of CNST is the greatest contribution doctors can make to improved Result, but three factors make this difficult. First, the presentation of clinical manifestations of the disease is not specific. Second, alternative diagnostic methods are deficiently estimated. Third, chemotherapy and neurosurgical intervention has been difficult to study.Clinical diagnosis of CNSTs cannot be made nor excluded on the basis of history and clinical assessment, although recall of late times exposure to tuberculosis can be beneficial. Chest imaging earlier pulmonary tuberculosis in many patients with CNSTs;

Corresponding Author: Mutasem Abuhamed, Department of Neurology, The First Xiangya Hospital, Central South University, Changsha410013, Hunan, People's Republic of China Tel: 0086-13787216436 Fax: 0086-0731-8634305 
American J. Infect. Dis., 4 (2): 168-173, 2008

therefore it is very helpful when it is shown by chest imaging studies.a high index of clinical suspicion is needed to diagnose CNSTs. In some patients, the attack can be subtle clinical symptoms that do not immediately propose the diagnosis; in others, the disease can present as intracranial masses. Given the fatal outcome of postponed treatment, clinicians should be supported to initiate empirical therapy in the setting of compatible clinical, imaging and laboratory findings. The presentations of clinical manifestations for the CNST have described extensively (Table 1$)^{[7-14]}$ the mechanisms that cause them are unintelligible. These mechanisms are important for doctors who need to understand the consequences of the disease and could lead to new treatments.

Neurological imperfection takes place with the progression of an inflammatory exudate, which principally affects the basal cisterns, sylvian fissures, brainstem and cerebellum. the process bring about most of the usual neurological deficits: The granulomas can coalesce to form CNSTs which, relying on their location, cause different clinical manifestation. The developments of CNSTs have been noted during the treatment of miliary tuberculosis or tuberculous meningitis while other tuberculous lesions have cured $^{[8,15]}$ the precise mechanisms of development of CNSTs are disputable. Possibly the expansion of the CNSTs has an immunological basis ${ }^{[16]}$. Some of the attention is that in some patients, one of the lesions seen an increase in size, while the other CNSTs declined during the same period of time, or in some patients some intracranial tuberculomas developed and enlarged at a stage when systemic tuberculosis was being treated successfully ${ }^{[7,15,17]}$. This supports the regard that local tissue reactivity is dependable for a multiplicity of different paradoxical responses in intra cranial tuberculomas $^{[2]}$. Infected hosts develop hypersensitivity to an array of mycobacterial proteins. Tuberculostatic drugs cause the destruction of mycobacterial structures and edit bacillar mycobacterial proteins, leading to the presence of inflammation and swelling of the of focus ${ }^{[8,18]}$. These provoke a delayed hypersensitivity reaction. The intracranial microtuberculomas grow slowly and become encapsulated after a latent period, resulting in paradoxical progression of existing lesions, which will be supported by an immunological accompanying phenomenon, namely the local perilesionally secondary granulomatous vasculitis associated with intimal proliferation and degeneration of the vessel wall with occlusion of the vessel lumen, which worsens the penetration of the tuberculostatic drugs into the lesions ${ }^{[8]}$.
Table 1: Multiple and solitary tuberculomas on presentation ${ }^{[7-14]}$

\begin{tabular}{|c|c|c|}
\hline Variables & $\begin{array}{l}\text { Multiple } \\
\text { tuberculomas }\end{array}$ & $\begin{array}{l}\text { Solitary } \\
\text { tuberculomas }\end{array}$ \\
\hline \multicolumn{3}{|l|}{ Symptoms } \\
\hline Meningeal irritation & ++ & + \\
\hline Headache & ++ & ++ \\
\hline Fever & ++ & + \\
\hline Vertigo & + & + \\
\hline Vomiting & ++ & + \\
\hline Anorexia & + & + \\
\hline Photophobia & + & + \\
\hline Increased lethargy & ++ & ++ \\
\hline \multicolumn{3}{|l|}{ Clinical signs } \\
\hline Neck stiffness & ++ & + \\
\hline Confusion & ++ & + \\
\hline Coma & + & + \\
\hline Cranial nerve III palsy & ++ & + \\
\hline Cranial nerve VI palsy & ++ & + \\
\hline Cranial nerve VII palsy & ++ & + \\
\hline Hemiparesis & ++ & + \\
\hline Seizures & + & ++ \\
\hline \multicolumn{3}{|l|}{ CSF } \\
\hline CSF glucose & - & - \\
\hline Appearance clear & - & - \\
\hline opening pressure & - & - \\
\hline Total leucocyte count & - & - \\
\hline Neutrophils & - & - \\
\hline Lymphocyte & - & - \\
\hline Protein & - & - \\
\hline Lactate & - & - \\
\hline \multicolumn{3}{|l|}{ Tuberculomas } \\
\hline Ophthalmologic symptoms & & $29.5 \%$ \\
\hline Intracranial hypertension & & $43.5 \%$ \\
\hline Optic nerve atrophy & & $18.5 \%$ \\
\hline Papillary oedema & & $43.7 \%$ \\
\hline Hemiplegia & & $16.8 \%$ \\
\hline Hemiparesis & & $30.5 \%$ \\
\hline Monoplegia & & $10.5 \%$ \\
\hline Aphasis & & $5.2 \%$ \\
\hline Cerebellar syndromes & & $23.2 \%$ \\
\hline Cranial nerves lesions & & $23.2 \%$ \\
\hline Epilepsy & & $28.4 \%$ \\
\hline Poor general state & & $9.5 \%$ \\
\hline Pulmonary tuberculosis & & $12.6 \%$ \\
\hline Lymph node tuberculosis & & $2.1 \%$ \\
\hline Vertebral tuberculosis & & $6.3 \%$ \\
\hline Tuberculin skin tests & & $70.3 \%$ \\
\hline Anaemia & & $17.4 \%$ \\
\hline
\end{tabular}

++: More common; +: Less common; -: Slight changes or normal

Magnetic Resonance Imaging (MRI) and Computed Tomography (CT) are the most frequent imaging techniques utilized in characterization and localization of CNSTs and provide diagnostic information at presentation. Although imaging manifestation are known to overlap with other intracranial focal lesions.

The radiological features of tuberculoma on CT scan vary according to its stage. Mature tuberculomas appear as a well-delineated round or oval ring enhancing mass with occasionally a target sign. The immature tuberculomas are iso- to hyperdense on the 
plain CT scan and show ring or nodular contrast enhancement following contrast administration. In many cases, solitary tuberculoma may be indistinguishable from abscess (pyogenic, fungal), tumor, cysticercus granuloma and other ring enhancing lesions on CT morphology alone. In these cases clinical, history, laboratory investigation, MRI and MR spectroscopy are helpful for differentiating these similar lesions ${ }^{[19-21]}$.

The MRI manifestations of a CNSTs rely on whether it is caseating with a solid centre, caseating with a liquid centre or noncaseating. The solid caseating granuloma gives isointense on T1- (Fig. 1A) and is isointense or hypointense on T2-weighted images (Fig. 1B $)^{[27]}$ The degree of decreased signal rely on the complex correlation between the presence of solid caseous material, macrophage infiltration, regional fibrosis or gliosis, macrophage byproducts and perilesional cellular infiltrate. The noncaseating granuloma usually gives hypointense than brain on T1and hyperintense on T2-weighted images, with homogeneous contrast enhancement. Physically, caseous material is cheesy in texture and is relatively high in lipid ${ }^{[22-24]}$ Lipid has been showed in caseating granulomas by in vivo proton. On in in vivo proton MR spectroscopy, lipids at $0.9 \mathrm{ppm}, 1.3 \mathrm{ppm}, 2.0 \mathrm{ppm}$ and $2.8 \mathrm{ppm}$ have been shown in tuberculomas that appear hypointense on T2 weighted images ${ }^{[8]}$. Recently, choline (3.22 ppm) has been observed along with lipids at $1.3 \mathrm{ppm}$ and $0.9 \mathrm{ppm}$ in a histologically cellular tuberculomas that showed mixed intensity on $\mathrm{T} 2$ weighted image. This resulted in its spectroscopic

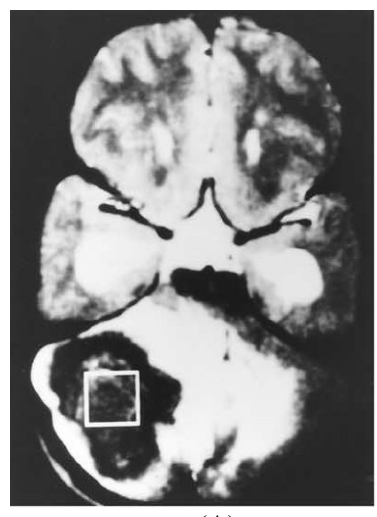

(A)

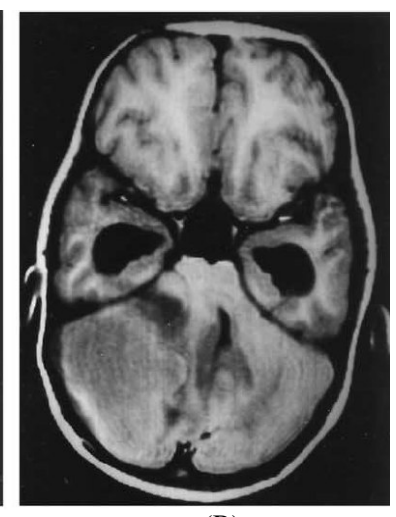

(B)
Fig. 1: T2 (A) and T1 (B) and images through the cerebellum show a large well-defined mass in the right cerebellar hemisphere predominantly hypointense on $\mathrm{T} 2$ weighted image (A) with mass effect and perifocal edema ${ }^{[27]}$ similarities with a neoplastic lesion ${ }^{[27]}$. The magnetization transfer MR imaging technique has recently accepted as an additional sequence to increase the value of image contrast and tissue specificity in various intracranial diseases ${ }^{[25-27]}$ number of researches have showed that the MT technique may be applicable in characterization of different Central nervous system lesions and quantitation of true disease load in various Central nervous system pathologies ${ }^{[25,27,28]}$. T1 weighted magnetization transfer MR imaging has been found to be applicable in differentiation of tuberculomas from cysticercus granuloma ${ }^{[23,27]}$. However, the $\mathrm{T} 1$ weighted magnetization transfers MR imaging is better than PMRS in tuberculomas showing higher cellularity and have mixed intensity appearance on conventional spin echo MR imaging. The both in vivo PMRS and T1 weighted MT MR imaging are useful techniques in better tissue characterization of intracranial tuberculoma. However, the T1 weighted MT MR imaging is superior to in vivo proton MR spectroscopy in tuberculomas showing higher cellularity and have mixed intensity appearance on conventional spin echo MR imaging ${ }^{[28]}$ Pathological changes include caseating granuloma with liquid centre, cascating granuloma with a solid centre andnon-caseating granuloma. MRI describes three sorts of tuberculoma pictures: tuberculous lesion seems isointense on T1WI and isointense to hypointense on T2WI and ring enhancement with lower signal centre on contrast MR $\operatorname{scan}^{[22]}$.

\section{BIOPSY}

Imaging Researches have failed to reliably diagnose CNSTs, necessitating histological confirmation. Biopsies show the histological changes of cerebral tuberculomas and give diagnostic information when complications occur. A final histological diagnosis before beginning anti tuberculosis treatment is suggested by many studies ${ }^{[29-31]}$, however, it has the defect of a major procedure with associated surgical risk. On the other hand, stereotactic biopsies, although they are slightly invasive and associated with insignificant risk, are often not resulting in a final conclusion $^{[32]}$. In a previously published works, stereotactic biopsies utilizing smear techniques for pathological diagnosis could dependably diagnose tuberculomas in only $28 \%$ of cases. In the rest, a chronic inflammation with gliosis was the histopathological picture ${ }^{[32]}$. However, Aaron Mohanty and colleagues ${ }^{[33]}$ found that paraffin sectioning and histopathological examination had an overall diagnostic efficacy of $85 \%$. 


\section{TREATMENT OF CNSTs}

The best treatment for CNSTs has been developed from the results of several studies ${ }^{[32]}$. The same is not true of CNSTs-choice of drugs, doses and duration of treatment are unknown and There are few data to guide the doctor. Nevertheless, there are frequent principles for medical treatment, extracted from the different roles of four antituberculosis drugs in the treatment of pulmonary tuberculosis; ethambutol and streptomycin kill most of the rapidly replicating bacilli in the first 14 days of treatment. Thereafter, Rifampicin kills low or non-replicating organisms and pyrazinamide kills those in sites hostile to the penetration and action of the other drugs. pyrazinamide and rifampicin sterilise lesions by killing organisms ${ }^{[37]}$. The treatment of Central nervous system tuberculoma is becoming more conservative. It consisted of four antituberculous antibiotics (Rifampicin 10-20 mg kg${ }^{-1}$ Oral 9-12 months, Isoniazid $10-15 \mathrm{mg} \mathrm{kg}^{-1}$ 9-12 months, Ethambutol 15-20 mg kg${ }^{-1}$ Oral 2 months Pyrazinamide $15-30 \mathrm{mg} \mathrm{kg}^{-1}$ Oral 2 months). The addition of fluoroquinolones may be of interest. the efficiency of steroids in reducing the perilesional oedema and threatening intracranial hypertension was observed ${ }^{[34-36]}$ Dexamethasone did not affect the site or the total numbers of tuberculomas or the proportion of patients with one or more tuberculoma. Tuberculomas developed in most patients during treatment, which suggests that they are part of the normal pathological response to treated infection ${ }^{[37]}$. The effect of dexamethasone on CNSTs is unclear. Therefore, dexamethasone might improve survival, but the anti-infl amatory mechanisms for doing so remain obscure. Other extracerebral eff ects of dexamethasone might be equally important. In particular, dexamethasone might protect against severe drugrelated liver toxicity and prevent life-threatening interruptions in anti-tuberculosis chemotherapy. Anticonvulsant treatment is mandatory for seizure control $^{[37]}$. Few patients with tuberculomas treated by surgery in the literature The most important cause of this is that most of the paradoxical tuberculomas (77\%) are deeply located lesions. Therefore, surgery of these lesions has a high risk. However, if a large lesion is not located in deep regions of the brain, it may be totally removed surgically. In addition, surgical treatment should be considered for patients with life-threatening neurological involvement and for patients with lesions that fail medical treatment ${ }^{[7,217,38]}$.

\section{CONCLUSION}

We would like to stress the following points (1) Central nervous system tuberculomas is uncommon, as is cerebral tuberculosis. Therefore, diagnosis should be kept in mind when confronted with intracranial masses; the diagnosis of CNSTs can be expedited by the presence of an extra cranial tuberculous lesion. Nevertheless, the diagnosis of CNSTs is less likely to be considered when the lesion is solitary; located in the brain and when laboratory and physical examinations are negative. (2) T1 weighted MT MR and in vivo PMRS Imaging are the best techniques in tissue characterization of CNSTs. (3) Swift, pharmaceutical and surgical treatment is recommended for the CNSTs. The most important follow-up criteria should be the MRI findings. (4) Surgical intervention in situation of acute complication of CNSTs, when the diagnosis is not shown to be true and there is no response to chemotherapy with in 8 weeks a biopsy should be performed.

\section{REFERENCES}

1. Dubé, M.P., P.D. Holtom and R.A. Larsen, 1992. Tuberculous meningitis in patients with and without human immunodeficiency virus Infection. Am. J. Med., 93: 520-524.

2. Thonell, L., S. Pendle and L. Stacks, 2000. Clinical and radiological features of South African patients with tuberculomas of the brain. Clin. Infect. Dis., 31: 619-620.

3. Martinez-Vazquez, C., J. Bordon, A. RodriguezGonzalez, J. de la Fuente-Aguado, B. Sopena, A. Gallego-Rivera and P. Martinez-Cueto, 1995. Cerebral tuberculoma: A comparative study in patients with and without HIV infection. Infection, 23: 149-153.

4. Zavascki, A.P., A.E. Dias, R.P. Cruz, R.L. de Oliveira and R.P. Duquia, 2006. Intracranial tuberculomas in an immunocompetent patient mimicking brain metastasis of unknown origin. Infection, 34: 181-182

5. Dolin, P.J., M.C. Ravlglione and A. Koehi, 1994. Global tuberculosis incidence and mortality during 1990-2000. Bull. World Health Organ., 72: 213-220.

6. Klein, N.C., B. Damsker and S.Z. Hirschman, 1985. Mycobacterial meningitis: Retrospective analysis from 1970-1983. Am. J. Med., 79: 29-34

7. Hejazi, N. and W. Hassler, 1997. Multiple intracranial tuberculomas with atypical response to tuberculostatic chemotherapy: Literature review and a case report. Acta Neurochir (Wien), 139: 194-202. 
8. Bas, N.S., F.K. Güzey, E. Emel, I. Alatas and B. Sel, 2005. Paradoxical intracranial tuberculoma requiring surgical treatment. Pediatr. Neurosurg., 41: 201-5.

9. Sahaiu-Srivastava, S. and B. Jones, 2008. Brainstem tuberculoma in the immunocompetent: Case report and literature review. Clin. Neurol. Neurosurg., 110: 302-304.

10. Huang, C.R., C.C. Lui, W.N. Chang, H.S. Wu and H.J. Chen, 1999. Neuroimages of disseminated neurotuberculosis: Report of one case. Clin. Imaging, 23: 218-222.

11. Guzel, A., M. Tatli, U. Aluclu and K. Yalcin, 2005. Intracranial multiple tuberculomas: 2 unusual cases. Surg. Neurol., 64: S109-S112.

12. Rebai, R., M.Z. Boudawara, K. Bahloul, I. Chabchoub, S. Chaari, T. Boudawara and H. Ben Mansour, 2001. Cavernous sinus tuberculoma: Diagnostic difficulties in a personal case. Surg. Neurol., 55: 372-375.

13. Tsai, M.H., Y.C. Huang and T.Y. Lin, 2004. Development of tuberculoma during therapy presenting as hemianopsia. Pediatr. Neurol., 31: 360-363.

14. Akhaddar, M.A., M. Lmejjati, A. Mansouri, N. El Abbadi and F. Bellakhdar, 2001. Intracerebral tuberculoma-Report of 115 cases, 5, $\mathrm{N} 1$ [DB/OL]. http://www.panarabneurosurgery. org.sa/journal/apr2001/Intracerebral_Tuberculoma. htm.

15. Chambers, S.T., W.A. Hendrickse, C. Record, P. Rudge and H. Smith, 1984. Paradoxical expansion of intracranial tuberculomas during chemotherapy. Lancet, 2: 181-183.

16. Smith, H., 1987. Paradoxical responses during the chemotherapy of tuberculosis. J. Infect., 15: 1-3.

17. Yamamoto, N., S. Kadoya, T. Nakamura, T. Emori, R. Kwak and G. Hirose, 1985. A case report of intracerebral tuberculoma during antituberculous therapy (abstract). No Shinkei Geka, 13: 1007-1011.

18. Teoh, R., M.J. Humphries and S.G. O'Mahony, 1987. Symptomatic intracranial tuberculoma developing during treatment of tuberculosis: A report of 10 patients and review of the literature. Q. J. Med., 63: 449-460.

19. Kastrup, O., I. Wanke and M. Maschke, 2005. Neuroimaging of infections. Neuro. Rx., 2: 324-332.

20. Gaviani, P., R.B. Schwartz, E.T. Hedley-Whyte, K.L. Ligon, A. Robicsek and P. Schaefer, 2005. Diffusion-weighted imaging of fungal cerebral infection. AJNR Am. J. Neuroradiol., 5: 1115-1121.
21. Kumar, R., M. Prakash and S. Jha, 2006. Paradoxical response to chemotherapyin neurotuberculosis. Pediatr. Neurosurg., 42: $214-222$

22. Poonnoose, S.I., S. Singh and V. Rajshekhar, 2004. Giant cerebellar tuberculoma mimicking a malignant tumour. Neuroradiology, 46: 136-139

23. Gupta, R.K., A. Jena, A. Sharma, D.K. Guha, S. Khushu and A.K. Gupta, 1988. MR imaging on intracranial tuberculomas. J. Comput. Assist. Tomogr., 12: 280-285

24. Wilson, J.D. and M. Castillo, 1994. Magnetic resonance imaging of granulomatous inflammations: Sarcoidosis and tuberculosis. Top Magn. Reson. Imaging, 6: 32-40

25. Venkatesh, S.K., R.K. Gupta, L. Paul, M. Husain and N. Husain, 2001. Spectroscopicincrease in signal is not specific marker for differentiation of infective/inflammatory from neoplastic lesions of the brain. J. Magn. Reson Imaging, 14: 8-15.

26. Boorstein, J.M., K.T. Wong, R.I. Grossman, L. Bolinger and J.C. McGowan, 1994. Metastatic lesions of the brain: Imaging with magnetization transfer. Radiology, 194: 799-803.

27. Gupta, R.K., M. Husain, D.K. Vatsal, R. Kumar, S. Chawla and N. Husain, 2002. Comparative evaluation of magnetization transfer MR imaging and in vivo proton MR spectroscopy in brain tuberculomas. Magn. Reson Imaging, 20: 375-381

28. Elster, A.D., J.C. King, V.P. Mathews and C.A. Hamilton, 1994. Cranial tissues: appearance at gadolinium-enhanced and non-enhanced MR imaging and magnetization transfer contrast. Radiology, 190: 541-546

29. Bouchama, A., M.Z. Atkawi, L. Kanaan, R. Coates, A. Jallu, B. Rabim and E.B. Siqueria, 1991. Brain biopsy in tuberculomas: The risks and benefits. Neurosurgery, 28: 405-409.

30. Rosenblum, M.L., 1991. Comments to brain biopsy in tuberculomas. Neurosurgery, 28: 409.

31. Selvapandian, S., V. Rajsekhar, M.J. Chandy and J. Idikula, 1994. Predictive value of computed tomography based diagnosis of intracranial tuberculomas. Neurosurgery, 35: 845-850.

32. Rajsekhar, V. and M. Chandy, 1993. CT-guided stereotactic surgery in the management of intracranial tuberculomas. Br. J. Neurosurg., 7: 665-671.

33. Mohanty, A., V. Santosh, B. Anandh, V.R. Kolluri, M.K. Vasudev, T. Hegde and S.K. Shankar, 1999. Diagnostic efficacy of stereotactic biopsies in intracranial tuberculomas. Surg. Neurol., 52: 252-258. 
34. Ghani, B. and J.M. Lieberman, 1994. Paradoxical enlargement or development of intracranial tuberculomas during therapy: Case report and review. Clin. Infect. Dis., 19: 1092-1099.

35. Kamura, M., E. Nakamura and S. Yoshii, 1986. Therapy effect of a new antibacterial substance ofloxacine (DL8280) on pulmonary tuberculosis. Am. Rev. Respir. Dis., 131: 352-356.

36. Addar, A., A. Bourazza, B. El Mostarchid, A. Rouimi, H. Ouahabi and R. Mossedaq, 2000. Tuberculomes du tronc cerebral. Deux case. Rev. Neurol., 156: 399-402.
37. Thwaites, G.E., J. Macmullen-Price, T.H. Tran, P.M. Pham, T.D. Nguyen, C.P. Simmons, N.J. White, T.H. Tran, D. Summers and J.J. Farrar, 2007. Serial MRI to determine the effect of dexamethasone on the cerebral pathology of tuberculous meningitis: An observational study. Lancet Neurol., 6: 230-236.

38. Bhargava, S. and P.N. Tandon, 1980. Intracranial tuberculoma: A CT study. Br. J. Radiol., 53: 935-945. 